\title{
ULTRA-DIELECTROPHORETIC FORCE SPECTROSCOPY ARRAY FOR PROBING INTERMOLECULAR AFFINITIES
}

\author{
Sam Emaminejad ${ }^{1,2,3 *}$, Salmonn Talebi ${ }^{2,3 *}$, Ali Javey ${ }^{l}$, Ronald W. Davis ${ }^{2,3}$, and Mehdi Javanmard ${ }^{4}$ \\ ${ }^{1}$ Department of Electrical Engineering and Computer Sciences, University of California, Berkeley, CA, USA \\ ${ }^{2}$ Stanford School of Medicine, Stanford, CA, USA \\ ${ }^{3}$ Stanford Genome Technology Center, Stanford University, Palo Alto, CA, USA \\ ${ }^{4}$ Department of Electrical and Computer Engineering, Rutgers University, Piscataway, NJ, USA \\ * Equal Contribution
}

\begin{abstract}
A multiplexed electronic force spectroscopy platform is demonstrated that can quantify the strength of the intermolecular interactions. As an example application, we demonstrate our platform's ability to characterize and differentiate specific and non-specific binding events.
\end{abstract}

\section{INTRODUCTION}

The ability to quantify the strength profile of the interbiomolecular bonds plays a significant role in various biomedical applications including biomarker detection, drug screening, and systems biology. Given the complex mechanisms and buffer conditions involved in determining the strength of the intermolecular bonds, development of a multiplexed force spectroscopy platform capable of probing a large number of interactions simultaneously and under the same buffer conditions is a necessity. The most common methods used to characterize such molecular interactions are atomic force microscopy, optical tweezers and magnetic tweezers. These techniques are limited in that they can only probe a single to a few interactions at a time. To this end, electronic-based actuation platforms are excellent candidates as they are scalable, addressable and provide precise control over applied voltage which can be programmed to exert various force profiles in order to characterize molecular interactions. The challenge with electrokinetic forces, such as dielectrophoresis lies in the inherent weakness of these forces (1$10 \mathrm{pN})$ compared to typical biomolecular interactions $(\sim \mathrm{nN})$. Previously, we enhanced the strength of the dielectrophoresis (DEP) force by two orders of magnitude (referred to as ultra-DEP, $\mathrm{uDEP}$ ) to overcome protein-protein interactions and to perform bead-based protein assays [1]. Here, we present a multiplexed uDEP-based platform that utilizes micron-sized beads as functional probes to examine intermolecular interactions and retrieve their force spectrums. As an example application, we demonstrate our platform's ability to characterize and differentiate specific and non-specific binding events.

\section{RESULTS AND DISCUSSION}

As shown in Figure 1, we patterned an array of proteins along a single microfluidic channel, where each element targets a secondary protein coated on micron-sized beads in the subsequently introduced sample. Below each element of the array, we have a pair of addressable interdigitated electrodes (IDEs). Through controlling the voltage applied at each IDE we can exert the desired force profiles on the beads and determine whether rupture of intermolecular bonds occurs (as evident by the bead detachment, observed optically). Here, we illustrate 16-plex actuation capability of our device to characterize the strength profile of anti-IgG/IgG (specific) and anti-IgG/IgM (non-specific) interactions. To this end, we functionalized the IDEs either with IgG or IgM, and used $2.8 \mu \mathrm{m}$-diameter anti-IgG coated beads to perform our pulling assay.

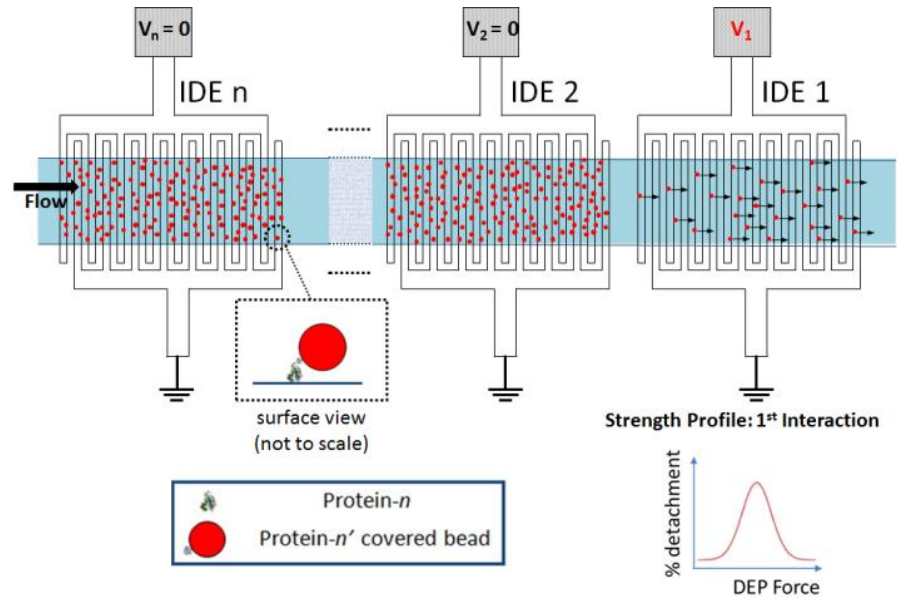

Figure 1: Ultra-dielectrophoretic force spectroscopy array for probing intermolecular interactions. An array of uDEP electrodes are patterned, each functionalized with a different protein that targets a secondary protein coated on the micron-sized beads. Detachment percentage of protein-bound beads is quantified optically when ramping up the applied voltage at each of the IDE pairs one-by-one. Here, $V_{1}$ is ramped up and the subsequently produced $u D E P$ force detaches the bound beads from the surface of the $1^{\text {st }}$ interdigitated electrode pair (i.e. IDE 1). With no voltage applied at the other IDEs, the protein-bound beads on the respective elements remain attached to the surface.

The two orders of magnitude of enhancement in DEP force (to overcome these interactions) was achieved by fabricating high voltage tolerant electrodes, In particular, a pinhole free nanometerscale thin film oxide as a protective layer was deposited using atomic layer deposition. The uDEP electrodes were excited at sufficiently high frequency $(1 \mathrm{MHz})$ to minimize the undesired voltage drop across the oxide film (Figure 2).

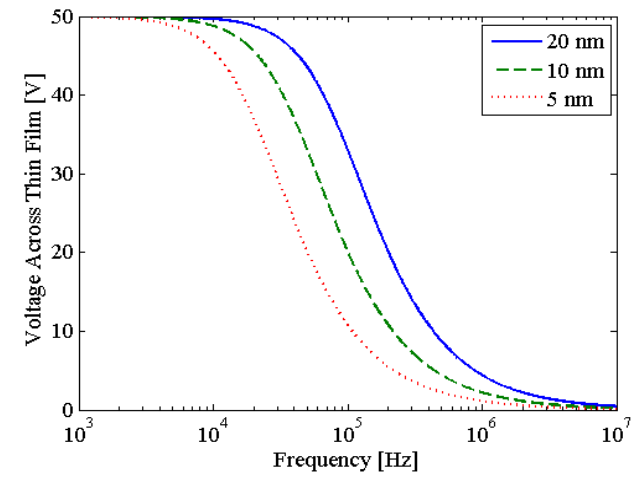

Figure 2: Voltage drop spectrum across the oxide capacitance at each electrode-buffer interface for various oxide thicknesses (simulated based on the developed circuit model). 
Representative four impedance spectrums of the IDEbuffer interface are shown in Figure 3 verifying that the resistive plateau region is achieved at $>1 \mathrm{MHz}$ (for $30 \times$ diluted PBS buffer).

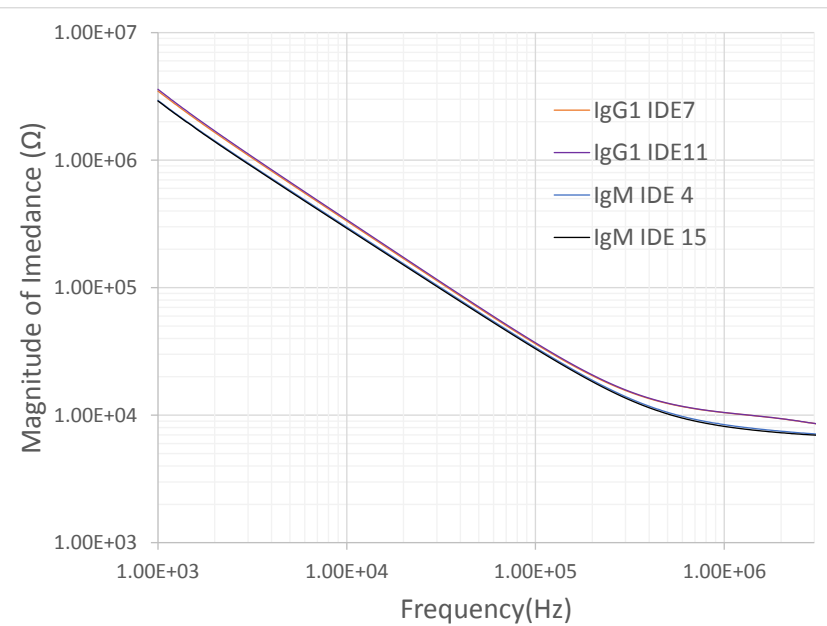

Figure 3: Measured impedance spectrum of four representative IDEs (IDE 7 and 11 are functionalized with IgG1 and IDE 4 and 15 are functionalized with $\operatorname{Ig} M$ ).

To retrieve the force spectrum for each interaction, the applied voltage was ramped up incrementally at each IDE. As can be seen in Figure 4, the peak of the anti-IgG/IgM bead detachment occurred when $12.5 \mathrm{~V}$ applied, while about 15 to $17.5 \mathrm{~V}$ was required to detach anti-IgG/IgG beads. These results indicate our platform's promising capability in distinguishing between specific and non-specific bindings.
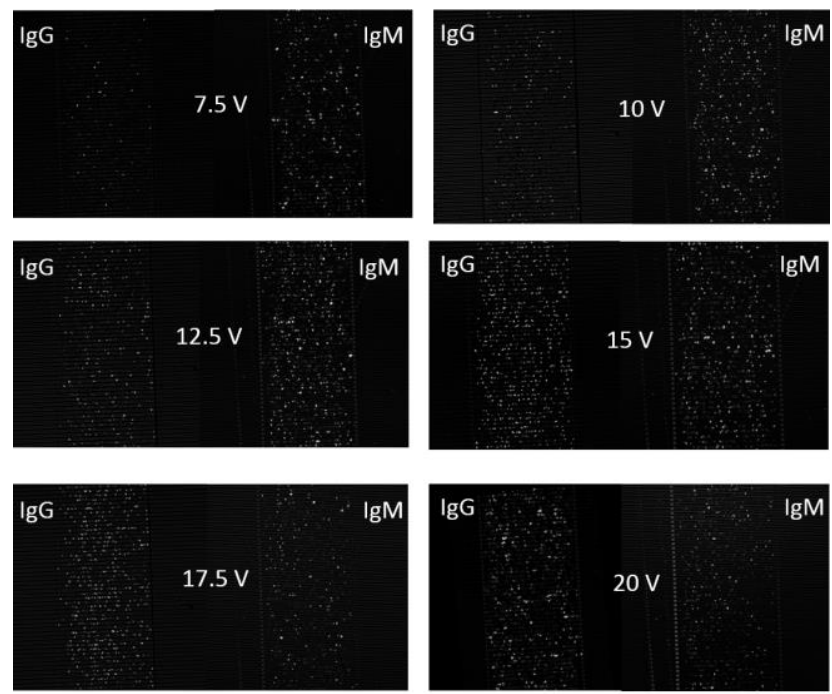

Figure 4: Post-processed (differential) and side-by-side micrographs of two representative IDEs, one originally coated with specifically bound beads (i.e. anti-IgG/IgG) and one originally coated with non-specifically bound beads (anti$\operatorname{Ig} G / \operatorname{IgM})$. In each frame the bright spots correspond to the detached beads.

The collective detachment profile when actuating anti$\mathrm{IgG} / \mathrm{IgG}$-bound beads vs. anti-IgG/IgM-bound beads is shown in Figure 5. This multiplexed format enables performing force spectroscopy on multiple devices independently, in a single channel; minimizing inter-experiment variations that originate from disparities in buffer conditions and assay preparation steps.

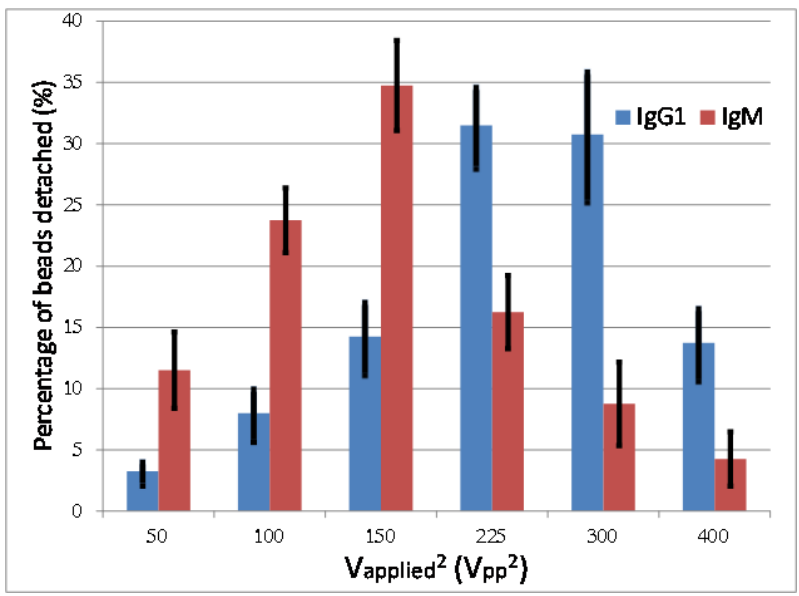

Figure 5: Detachment percentage of protein-bound beads when ramping up the applied voltage at each of the IDE pairs one-byone. The results depict the collective detachment profile when actuating specifically- (anti-IgG/IgG) vs. non-specifically- (antiIgG/IgM) bound beads using our 16-plex platform. The array format allows performing force spectroscopy multiple times while ensuring operation under the same buffer conditions and assay preparation steps (DEP force is proportional to the square of the applied voltage).

We envision a plethora of possibilities for performing rapid and inexpensive characterization of intermolecular affinities which is of utmost importance in studying various inter- and intracellular molecular pathways.

\section{ACKNOWLEDGEMENTS}

This work was supported by the National Institutes of Health through grant PO1HG000205. Fabrication of the devices was performed in the Stanford Nanofabrication Facility.

\section{REFERENCES}

[1] S. Emaminejad, R.W. Dutton, R.W. Davis, and M. Javanmmard, "Multiplexed Actuation Using Ultra Dielectrophoresis for Proteomics Applications: A Comprehensive Electrical and Electrothermal Design Methodology", Lab Chip, 14 (12), 2105-2114 (2014)

\section{CONTACT}

*S. Emaminejad, sam.e@berkeley.edu 